\title{
A Novel Filtered Segmentation-Based Bayesian Deep Neural Network Framework on Large Diabetic Retinopathy Databases
}

\author{
Shaik Akbar ${ }^{1 *}$, Divya Midhunchakkaravarthy ${ }^{2}$ \\ ${ }^{1}$ Department of Computer Science \& Engineering, Lincoln University College, Selanger 47301, Malaysia \\ ${ }^{2}$ Department of Computer Science \& Engineering, Head Academic and Student Affairs, Centre of Post Graduate Studies, \\ Lincoln University College, Selanger 47301, Malaysia
}

Corresponding Author Email: dr.akbar@pscmr.ac.in

https://doi.org/10.18280/ria.340602

Received: 24 August 2020

Accepted: 10 December 2020

Keywords:

diabetic retinopathy, feature ranking,

Bayesian classification, deep neural network

\begin{abstract}
Image thresholding-based segmentation models play a vital role in the detection of Diabetic retinopathy (DR) on large databases. Most of the conventional segmentation-based classification models are independent of over segmented regions and outliers. Also, these models have less true positive rate and high error rate on different DR feature sets. In order to overcome these problems, a novel filtered based segmentation framework is designed and implemented on the large DR feature space. In this work, a novel image filtering approach, optimal image segmentation approach and hybrid Bayesian deep learning framework are developed on the large DR image databases. Experimental results proved that the proposed filtered segmentation-based Bayesian deep neural network has better accuracy and runtime than the conventional models on different DR variation databases.
\end{abstract}

\section{INTRODUCTION}

Diabetic retinopathy is eye disease that causes retinal nerve hemorrhage in the eye that indicates loss of vision. The small blood vessels of the retina damage retinopathy, which causes a significant loss of view. It is estimated by the National Eyo Institute that $40 \%$ to $45 \%$ of American diabetics become blind every year by diabetes retinopathy [1]. Diabetes retinopathy symptoms do not appear until the retinal vision, normally by partial vision, has been visually damaged. Consistent eye screening is therefore necessary for early diagnosis and treatment before significant damage to the retina is caused because the risk of blindness in these patients may be reduced by $50 \%$. Early detection of DR allows laser therapy to stop or delay visual loss and can help improve the diabetic control. An automatic diabetic retinopathy detection and therapy at an early stage can thus prevent blindness [2]. Caused mostly for diabetic patients, retinopathy is diabetic. For the extraction of diabetic retinopathy diseases, this study makes image segmentation [3]. Diabetes is a disease in which glucose metabolism abnormalities lead to increased blood glucose levels. One of the prominent complications caused by prolonged diabetes is diabetic retinopathy [4]. The percentage of globally diabetes-affected population is growing at an alarming rate. The global prevalence of diabetes is expected to rise from 130 million to 300 million in the next two decades, according to World Health Organization reports [5] Prolonged diabetes affects the retinal vasculature and ultimately results in Diabetic Retinopathy (DR) and subsequent vision loss. DR complications and subsequent blindness can be moderated if periodic screenings detect the DR early enough. According to UK surveys, early detection of DR by periodic diabetic patient retinal screening may reduce the risk of blindness in these patients by $50 \%$ [6]. The potential ability of computerized algorithms and automated systems to show diabetic retinopathy or absence during periodic screening programs

High dimensionality is a major issue where a large number of training features are processed to perform mathematical operations such as transformation of data, classification of data, etc. Traditional dimensionality reduction techniques are implemented using the same and defined number of features to reduce the number of dimensions. Ensemble classification models are used to predict the high dimensional features with less error rate in the specified training datasets. Through learning model of an ensemble combines multiple base classifiers to boost the accuracy compared to its individual prediction score.

Artificial neural network typically has three layered architecture for data classification, such as input layer, hidden layer, and output layer. Scientists have made tremendous efforts in recent years to improve the architecture of networks and learning models based on basic ANN [7]. But in the neural network setting, the major challenge is choosing the correct activation function using the logistic and hyperbolic functions. Since the proper activation function selection improves the classification rate on the high dimensional datasets.

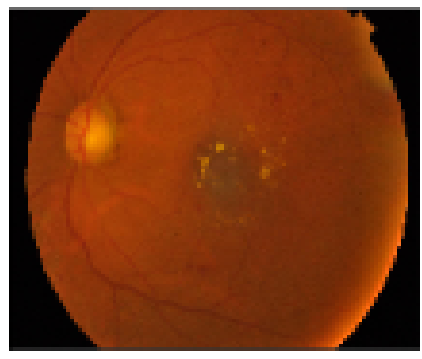

Figure 1. Different variation in diabetic retinopathy image for classification 
To address the dimensionality and error rate of data in conventional selection and classification models, large and heterogeneous datasets are treated with feature selection based ensemble classification. Using the novel optimization functions, this optimal feature selection model is used to solve complex patterns. The main goal of this model is to increase the true positive rate of classification by reducing the error rate using the measure of hybrid feature selection as shown in Figure 1. Precise location of the boundary of the optical disc is a major sub-problem for the processing of ophthalmic images.

The location of the optical disk is a major challenge in retinal image analysis as the location of anatomic components in retinal images, vessel tracking and registering changes due to disease and size within the optical discography area is a key feature. To distinguish the disk from the other features of the retina, it is often necessary to located the disk. Fixed position of the optical disk may improve the removal of the disk boundary. The location of the optical disk is a prerequisite for determining certain important vasculature-oriented diagnostic indexes of hypertensive or sclerotic retinopathy [8].

Digital retinal imaging is a stimulating method of screening which still needs to establish effective, robust and costefficient methods. In order to identify the risk group for visual damage, a consistent screening of diabetic retinopathy and diabetic maculopathy diseases is essential [9]

Image segmentation walls of the image into different areas by making the image more readable and understandable. The process of segmentation applied to different image types. Segmentation technique plays a major role in the use of retina images to help early diagnosis of persuaded eye-related illness. Correct retinal blood vessel segmentation plays a very prominent role in diagnosing vascular retinal diseases. The thresholding on an entropy basis is proposed with several features. Several characteristics are extracted, such as vessel measurement, Gabor filter magnitude response and Gamma correction with inverted green canal. Collective features with neural network are extracted. The segmented blood vessel is then used to obtain the Entropy-based threshold. Porwal et al. [10] presented a new method by three steps to detect bleeding, eliminate noise from the backdrop image, remove vessels, remove fovea form, area, aspect ratio, density and mean intensity. A new vessel filter to distinguish between red lesions and blood vessels is proposed to address false detections.

The prominent cause of blindness is diabetic retinopathy. According to recent health studies, 54 million Americans have diabetes and most don't know it. WHO reveals this worldwide diabetes to about 347 million people every year, up to 25,000 people lose sight of their cause of diabetic retinopathy [11]. The retinal micro vasculature causes diabetic retinopathy. Unchecked and severe cases of diabetic retinopathy may result in blindness. A highly time-consuming and recurring study of fundus pictures to check morphologic changes in microaneurysms, exudate material, blood vessels, hemorrhages and macula [12].

Compared to the retinal disease problem in videos, the retinal detection problem in still images is more compelling and difficult due to large number of feature space. Various eye diseases have distinctive side effects that lead to disease recognition. The retinal vein impediment shows the indication that the retinal artery occlusion has changed the shades of the conduits that are shading copper or silver. Diabetes is a disease that affects parts of the body such as the kidneys, eye, sensory system, heart, etc. The human eye, which is worthy of mention of the human body, needs special attention as it affects the vision. The ill effects of glaucoma, DR, cataracts, occlusions in the retinal arteries and occlusions in the retina 's veins may be experienced by those with diabetes. The authors calculated the vessel's boundaries, track the midline of the vessel, and concentrate valuable clinical value parameters according to the coordinated filter method combined with earlier information on retro vascular blood vessel characteristics. By showing the ship profile that uses Gaussian capacities, they are given better assessments of ship widths by developing new algae in past calculations, but not so much variation could be found [13].

Iris recognition system comprises a process in which iris is located and iris images are generated and their motifs are recognized. For the location of internal and external boundaries of the iris area a faster algorithm is applied. A neural network (NN) is used with this data set to classify iris patterns, and a training set uses $\mathrm{CNN}$. The segmentation of iris took place here in a short time. The focus of this paper is mainly on the detection by thermal images of diabetic retinopathy. An algorithm known in particular as a Convolution Neural Network is used for this system. CNN is the deep learning branch that has various records for image analysis applications, including medical imaging. normalization and clinical diagnostic feature classification. For training and classification, the artificial neural network. Enhancement is essential for deep layer characteristics in the analysis of functions. A range of image enhancement methods have been applied for feature extraction such as arithmetical operation, histogram equalization and histogram equalization. This approach is useful in the fast, user-friendly, time consuming diagnostic field.

\section{RELATED WORKS}

Sambyal et al. suggests the presence of abnormalities throughout retina, such as blood vessel formation, microaneurysms and exudates, using image processing technique [14]. Using the Fuzzy C-Means Clustering Algorithm various types of diabetic retinopathy are observed. DR screening process allows identifying the signs more rapidly and conveniently. These modules perform segmentation of the image that includes segmentation of the vessels, exudates, MA and others to perform classification of fuzzy c-means. This gives diabetic retinopathy sensitivity, precision, and accuracy. Diabetic Retinopathy is a medical condition in which the retina is scratched because of fluid leaks in the retina from the blood vessels. Hemorrhages of the eye are the first signs of diabetic retinopathy.

Selçuk and Alkan [15] suggests a mixture of interior components of microaneurysm detectors, specifically preprocessing methods and applicant extractors as microaneurysm detection is essential in the grading of diabetic retinopathy.

Shankar et al. [16] explains the identification of microaneurysms in wireless fundus snapshooting, which is considered a very important method of detection for automatic diabetic retinopathy screening. In the worldwide microaneurysm identification for the Retinopathy Online Challenge, regular methods are required to complete the reconnaissance process. To generate a comparable set of data for further review the tests were compared with the different sets. The test is conducted in the training images, with appropriate actions for the data collection. 
Stolte and Fang [17] present in retinal picture to detect microaneurysms to determine the stage of the disease. Blindness-related conditions may be identified at an early stage by detecting Microaneurysms, which in effect helps to reduce DR growth. The proposed method helps to diagnose the symptoms quicker and also helps to detect MA even with low image quality. With the vast number of fundus images being processed efficiently, the cost is significantly reduced resulting in profitability and performance improvement. This requires no intervention from the user and can perform well in both normal and abnormal images. However, if there are a large number of retinal images it becomes a failure.

Waqar et al. [18] proposes an algorithm for calculating the thickness of retinal vessels in fundus photographs using a graph-based algorithm for sectioning both edges of the vessel at the same time. The graph-based algorithm isn't robust for a severe eye disease attack.

Washburn et al. [19] describe a technique based on active contours and mathematical morphology for identifying the boundary of OD in the fundus image of the retina. Preprocessing of the images is highly needed for effective boundary detection, which is based on local minima detection and morphologic filtering. Using active contour, the OD boundary is identified after pre-processing. This method has low stable yet reliable handling of high variable data.

Xie et al. [20] introduce a novel approach based on models for segmenting the optic disk from optical retinal images. This approach employs morphological and boundary identification methods followed by the Circular Hough Transform to achieve a rough estimate of spherical OD boundaries. Nevertheless, I need more pixels, put as preliminary knowledge within the OD.

Xie et al. [21] present in fundus images the original deformable-model-based algorithm for the complete mechanized recognition of the optical disk boundaries. The changes made for occlusions of the blood vessels in the model based algorithm are not quite reliable and stable.

You et al. [22] discusses the process of matching images of the same retina in order to study the development of certain pathologies. Genetic algorithm, in particular, is used to locate and recognize numbers on a license plate with a novel method for optimizing the overlapping process of various images acquired at different times, given that the images differ due to different acquisitions, different illustration and different pathology steps.

Amin et al. [23] was presented as the method for measuring the periodicity of components based on the classification of diabetic retinopathy. A non-invasive transient visual evoked period-based measurement method based on the potential of phase spectral components for the classification of diabetic retinopathy depends on spatial illumination variations resulting from the instrument limits, changes both within and among patient visits. A new iterative algorithm for estimating the reflectivity of the retina exploits retinal vasculature, optical disk, fovea and pathology automatically extracted segmentation. The resilience of dust artifacts can be achieved through the application of their spectral properties to filmbased and digital imagery systems.

de la Torre et al. [24] proposed a content-based image recovery method for diagnostic aids to diabetic retinopathy, with the use of a transformation of a 12 parameter that accounts for unknown retinal curvature and camera parameters. They proposed ridge-based boat segmentation on limited databases. The color of the retinal image work depended on the extraction of the image rim, \& coincided with the blood vessel center line. They were using k-NN classifiers to distinguish diseased and unknown images. But for higherlevel observers (more than second) the correctness of their method was not discussed. Their method did not provide information about the success of their methods even though they had good results for the selected database, w.r.t. other available bases were not available. One of the problems in their work was only the 2 nd order derivative functions, but the higher order derivatives were not satisfied.

Kamble and Kokate [25] presented a concise audit of the imaging techniques to detect the DR disease and its effects on severity together with its rating techniques in 33. This paper reviewed the different strategies for DR identifying and grouping into different phases depending on the limb level, and different DB images were also presented for the conduct of the research. They worked on the problems of classification, i.e., automatic detection and analysis of DR on the multi-class vector support machine. Although it gave very good results, its classification could not be satisfied if other classifiers were used for the same series of images from standard databases. The 2-D Gabor wavelet extracting the diabetic retinopathy lesions is used to classify the SVM. Analyzes of the retinal image with mobile phones are also addressed as a future trend in this area. A system for the analysis and exudation of micro aneurysms is being developed by the authors. Differences in the collected fundus image can be detected using morphological methods for digital image processing. Diabetic retinopathy with accuracy by 94 per cent is detected using the extracted features. Morphological procedures help detect anomalous traits of diabetic retinopathy by removing normal features of the retinal image. A novel algorithm is proposed to diagnose diabetic retinopathy by the detection of exudates using mathematical morphology methods to remove optic disks, macula, and blood vessels from the retinal fundus image. There are no early symptoms of diabetic retinopathy until the individual is excessive. The lens' eye fundus encompasses the retina and optic disk as well as the macula and fovea and the posterior poles. The initial phase of detection of diabetic retinopathy-patients and the second phase evaluate the extent to which the patient was pretentious. A diabetic diagnosis of diabetes retinopathy in fundus imaging using image processing technologies is a new method for red spot diagnostics, bleeding, detection of venal artery crossover points, input on color, form, size, and the disease detection. In the Disease Classifier stage, Kumar et al. [26] used a more robust technique which provides better performance and flexibility for distinguishing between candidate lesions and other information. Blood vessel segmentation based upon Gabor wavelet is used to diagnose many eye diseases in retinal images using kernel classification methods. The Kernel classifiers such as SVM and the relevance vector machine are used to segment the vessels by classifying every pixel vector by measuring the pixel intensity and measuring the Gabor wavelet responses at different scales. In terms of the specificity and sensitivity, the efficiency of the segmentation will be analyzed.

Liu et al. [27] use texture, threshold and morphology operations on retinal images. A robust, combined blood tree segmentation method on a $2 \mathrm{D}$ picture. In this work, the image and color contrast improvement are done in the preprocessing step and the combined approach is followed by texture, threshold and morphological functions for segmentation and classification of images. The A number of fundus pictures with different views and intensities are used to test this procedure. 
Shanthi and Sabeenian [28] discusses the segmentation of retinal vessels using regularizing multiconcavity models. Detecting blood vessels with bright and dark lesions in retinal images is a difficult, unresolved problem. The idea is to handle both healthy and unhealthy retinos simultaneously by a novel multiconcavity modeling approach. The measurement of differing concavity is proposed for treating bright lesions in a perceptive area. The concavity measurement of line forms is proposed to remove dark lesions with an intensity structure different from the linear vessels in a retina. SVM is a new statistical learning technique that was successfully used for pattern recognition applications, such as face and speaker recognition, and HMM is a potent statistical technology used to detect signatures and checks by handwriting. A suited study and testing protocol are designed to observe the classifiers' ability in random, simpler and stimulated forging to absorb interpersonal variability and highlight interpersonal similarity. It is worthwhile for this algorithm to require features smaller than standard methods. It appears in a gray fundus as a luminous white area and has a near round shape which is disturbed by the outbound vessels. The small but unmissable angle between the image planes and the object makes optical disk dimensions and sizes elliptical. Optical disks vary between patients. The segmentation of the optical disk is carried out using a modified transforming curve and after morphological transformation operation. Diabetic retinopathy is an eye disease that affects 80 to $85 \%$ of diabetes-patients over ten years. The images of retinal fundus are normally used in clinics to detect and analyze diabetic retinopathy. This work is carried out using the extraction of the green channel, histogram equalization, image enhancement and resize techniques to pre-process the raw retinal fundus images. 14 characteristics for quantitative analysis are also extracted from pre-processed images. The experiments are conducted with Kaggle Diabetic Retinopathy and the results are evaluated by taking into account the mean value for extracted functions and the standard deviation. In order to evaluate intensity variance of pixels, Wang et al. [29] used an $80 \times 80$ pixel substratum. In the assumption that visible signs of disease like exudates will show a low variance in intensity next to that of optic disk, the point with the highest variation in intensity was identified as optical disc. The red areas were successively broken into using the iterative region-growing technique and then a neural network was used to segment the blood vessels into hemorrhages within the retinal region.

\section{PROPOSED MODEL}

As shown in the Figure 2, image thresholding is a hybrid method which is used to find the essential key regions for the image segmentation process. In this work, a hybrid image thresholding method is developed by using the background and foreground regions for segmentation process. In this work, a hybrid image segmentation method is developed by using the kernel density estimation. Segmentation is a vital step in the development of DR automated diagnostic systems. In many algorithms, threshold-based segmentation is a key preprocessing component for automatically identifying other features. Segmentation is essential to set a reference framework within the retinal image and is therefore important for any application to analyze the image. Many optical disk localization schemes were proposed. Most of the techniques proposed to detect the optical disk focused on location and did not solve the problem of optical disco contour detection. Binary image was achieved by threshing the intensity image, including all bright regions. These features are used to find and classify the disease pattern on large number of training samples using deep learning-based classification model.

Proposed model is designed and implemented in two phases, i.e. image feature segmentation and filter based Bayesian deep neural network for disease severity classification. In the first phase, a novel mathematical filter is applied on the fundus training detect the shape and statistical analysis for disease severity classification. In this phase, a novel filtering approach is used to remove the noise and to smooth dark red dots and bright spot lesions. In the second phase, a deep CNN network is applied on the filtered data to find the essential features for classification problem. In the deep learning phase, a pretrained C3D framework is used to filter the essential features in each training image for classification problem. Finally, in the classification phase, a new classifier is implemented to predict the disease classes. In this phase, a hybrid non-linear Bayesian SVM classifier is used to train the C3D features for disease class prediction.

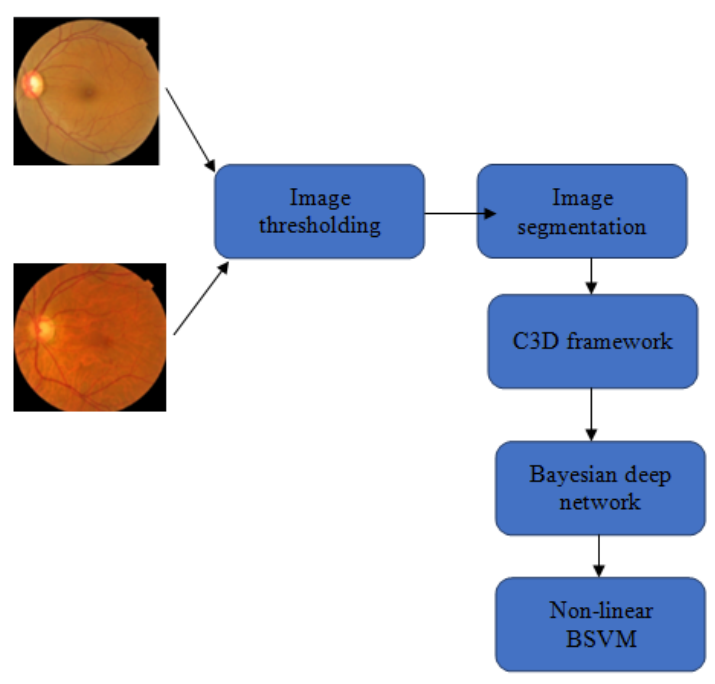

Figure 2. Proposed segmentation based Bayesian deep neural network

Step 1: Threshold feature segmentation

Step 2: Input training disease and normal images.

Step 3: Filter the images using disease patterns as.

Step 4: Determine the foreground level lower threshold using C-V model.

The variance of foreground level $f l=\{0,1,2 \ldots T h\}$ is

$$
\begin{aligned}
& G_{\min }(p, q) \\
& =\min \left\{\log (\lambda) \cdot \frac{e^{-\sqrt{p^{2}+p^{2}} / 2 \cdot \sigma_{p}^{2}}}{\sigma_{p} \sqrt{2 \cdot \pi}}, \log (\eta) \cdot \frac{e^{-\sqrt{p^{2}+q^{2}} / 2 \cdot \sigma_{q}^{2}}}{\sigma_{q} \sqrt{2 \cdot \pi}}\right\} \\
& G_{\max }(p, q) \\
& =\max \left\{\log (\lambda) \cdot \frac{e^{-\sqrt{p^{2}+p^{2}} / 2 \cdot \sigma_{p}^{2}}}{\sigma_{p} \sqrt{2 \cdot \pi}}, \log (\eta) \cdot \frac{e^{-\sqrt{p^{2}+q^{2}} / 2 \cdot \sigma_{q}^{2}}}{\sigma_{q} \sqrt{2 \cdot \pi}}\right\} \\
& T_{1}=(\max \{\lambda, \eta\}) \cdot * \sum \min \left\{p * \operatorname{Pr}\left((p, q) / c_{1}\right), q\right. \\
& \left.* \operatorname{prob}\left((p, q) / c_{2}\right)\right\} /|N| \\
& T_{1}=(\min \{\lambda, \eta\}) \cdot * \max \left\{p * \operatorname{Pr}\left((p, q) / c_{1}\right), q\right. \\
& \left.* \operatorname{prob}\left((p, q) / c_{2}\right)\right\} /|N|
\end{aligned}
$$


Step 5: Each disease intensity feature values of ROI and kernel density estimation are marked for feature extraction.

$$
\begin{gathered}
G(p, q)=e^{\left\{-\left(\frac{p_{1}^{2}}{\sigma_{p}^{2}}+\frac{q_{1}^{2}}{\sigma_{q}^{2}}\right)\right\}} \log \left(\frac{2 \pi p_{1}}{\lambda}\right) \\
\text { where, } \mathrm{p}_{1}=q_{1}=p \cos \phi+q \sin \phi \text { and } \sigma_{p}=\sigma_{q}=\sigma \\
G D(p, q)=\exp \left(\frac{2 p_{1}^{2}}{\sigma^{2}}\right) \cos \left(\max \left\{T_{1} \cdot \sigma_{p}, T_{2} \cdot \sigma_{q}\right\} \cdot\left(\frac{2 \pi q_{1}}{\lambda}\right)\right. \\
F S\left(G D(p, q), C_{m}\right)=\operatorname{Max}\left\{\frac{\operatorname{Prob}\left(G D(p, q) / C_{m}\right)}{|G D(p, q)| \cdot \operatorname{Prob}\left(C_{m}\right)}\right\} ;, m \\
=1,2 \ldots|G|
\end{gathered}
$$

Each image is partitioned into blocks to find the nearest neighbour features along with its variations. Let $B_{i}$ and $B_{j}$, represents the block partitions with means and covariance matrices of blocks ith and jth blocks of frame. In this step, each block and its adjacent blocks are used to find and mark the disease patterns and its orientations in each image.

\section{FEATURE EXTRACTION AND CLASSIFICATION USING C3D FRAMEWORK}

Most of the traditional C3D framework use convolution kernels of $3 \times 3 \times 3$ to filter the features in the disease prediction. Proposed C3D deep learning network is used to find the low-level features and to filter the ranked features obtained in the algorithm 1. Here, different convolution layers, max pooling and filters are used to find the essential features for data classification process. At the end fully connected layer is used to filter the essential disease features in the image using softmax activation function as shown in Figure 3. These features are used to classify the disease severity using the proposed classification model.

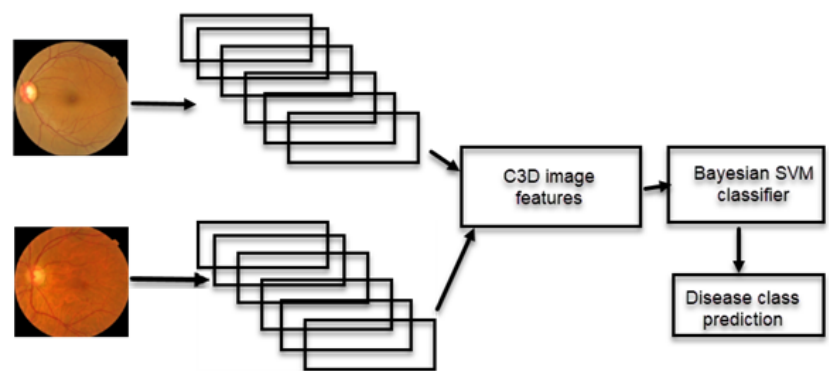

C3D framework

Figure 3. Segmentation based Bayesian Non-linear SVM model

\section{Algorithm:}

Adaptive Image Bayesian Neural Deep Network (ABNDN)

1. To each block B(i) in I

2. Do

3. Computing the normalized Gussian kernel on block B(i)

4. $G(B(i), B(j), \sigma)=\frac{1}{2 \pi \sigma_{B}{ }^{2}} \exp \left(-\frac{i^{2}+j^{2}}{2 \sigma_{B}{ }^{2}}\right)$

5 . The angular variation of the block can be determined by using
6. $A D G=\tan ^{-1}\left(\frac{B_{j}}{B_{i}}\right) \in(0,2 \pi]$

7. Compute the normalized kernel estimator NK(B): = $\frac{(G(B(i), B(j), \sigma)-A D G(B(i), B(j))}{}$

8. End

\section{Segmentation based C3D Classification model}

Proposed classification model is used to predict the severity level of disease based on the C3D features. Initially, deep learning features are trained using the feature extraction algorithm and then these features are filtered by C3D model based on class severity. A novel non-linear multi-class classification model is proposed to predict the level of disease severity on the selected features space.

Step:1: Feature space FS $=\mathrm{C} 3 \mathrm{DF}$ (Filter(Images));

Step:2: To each C3D feature $\mathrm{f}$ in FS

Step:3 Find the feature correlation based on the mutual information and chisquare measure as

$$
\begin{aligned}
& e_{\mathrm{k}}=\frac{1}{2} \text { correlation }\left(\mathrm{F}_{0}(\mathrm{r})\right) \cdot \sum_{a}^{m}\left(\mathrm{MI}_{0}(\mathrm{r})-O_{o}(\mathrm{r})\right)^{2} \\
& \mathrm{O}_{\mathrm{o}}(\mathrm{r})=\sum_{\mathrm{k}}^{\mathrm{L}} \mathrm{MI}_{\mathrm{ho}}(\mathrm{r}) \psi_{\mathrm{h} 0} \\
& \mathrm{~N}_{h o}(\mathrm{r})=\chi\left(\mathrm{N}_{h o}(\mathrm{r})\right) \cdot g\left(\mathrm{~N}_{i h}(\mathrm{r})\right. \\
& \mathrm{N}_{i h}(\mathrm{r})=\sum^{m} w_{i h} x_{i}(\mathrm{r})+\mathrm{a}_{h}
\end{aligned}
$$

Step 4: done

Step 5: Apply the non-linear SVM classification on the features using the following objective function and kernel function as

Minimization of the second objective function is represented as

$$
\operatorname{Min}\left\{\mathrm{O}_{1}\right\}=\omega \cdot \sum_{\mathrm{i}=1}^{\mathrm{k}} \sum_{\mathrm{j}=1}^{|\mathrm{RD}|} \mathrm{e}^{-\log \left|\mathrm{P}_{\mathrm{ij}}\right|} \cdot \mathrm{D}\left(\mathrm{g}_{\mathrm{i}}, \operatorname{Tr}[\mathrm{j}]\right)
$$

where, $\mathrm{D}$ is Euclidean distance.

$$
\begin{aligned}
& \min J=\frac{1}{2}\left\|\operatorname{sign}\left(w^{*} \varphi(x)_{i}+k\right)\right\|^{2} .+\operatorname{Max}\left\{O_{1}\right\}=\omega \cdot \sum_{i=1}^{k} \sum_{j=1}^{|R D|} e^{-\log \left|P_{i j}\right|} \cdot \log \left|P_{i j}\right| \\
& +\varepsilon . v_{\mathrm{t}} \sum_{\mathrm{i}=1}^{\mathrm{n}}\left(\gamma_{\mathrm{i}}^{\prime}+\gamma_{\mathrm{i}}\right) \\
& \text { s.t. } \\
& P_{i j}=\frac{\operatorname{Prob}\left(g_{i} / \operatorname{Tr}[j]\right) \cdot e^{-\left(\frac{D\left(g_{i}, \operatorname{Tr}[j]\right.}{\min \left(\left|G_{t}\right|,|, R D|\right)}\right)}}{\sum_{m=1}^{k} e^{-\left(\frac{D\left(g_{i}, \operatorname{Tr}[j]\right.}{m^{2} T_{r c}}\right)}} \\
& \text { where } g_{i} \in G_{t}, \operatorname{Tr}[i] \in R D, k \text { : number of segments. } \\
& \mathrm{T}_{\mathrm{rc}}=\sum_{\mathrm{i}=1}^{|\mathrm{Tr}|} \mathrm{g}_{\mathrm{i}} \cdot \operatorname{Tr}[\mathrm{i}] / \sum_{\mathrm{j}=1}^{|\mathrm{Tr}|} \mathrm{g}_{\mathrm{j}} \\
& v_{\mathrm{t}}=\frac{1}{2} \ln \left(\frac{1-\gamma_{\mathrm{t}}}{\gamma_{\mathrm{t}}}\right) \\
& \varepsilon=\underset{\mathrm{t} \in 1, \mathrm{M}_{1}}{\arg \min } v_{\mathrm{t}} \\
& \mathrm{w} \cdot \varphi(\mathrm{x})+\mathrm{k}-\mathrm{y}_{\mathrm{i}}-v_{\mathrm{t}} \leq \varepsilon+\gamma_{\mathrm{i}}^{*} \\
& \left(\mathrm{y}_{\mathrm{i}}-\mathrm{w} \cdot \varphi(\mathrm{x})-\mathrm{k}+v_{\mathrm{t}} \leq \varepsilon+\gamma_{\mathrm{i}}\right. \\
& \gamma_{\mathrm{i}}, v_{\mathrm{t}}, \gamma_{\mathrm{i}}^{\prime} \geq 0, \mathrm{i}=1,2, . . \mathrm{n}
\end{aligned}
$$




\section{EXPERIMENT RESULTS}

Experimental results are simulated in python and java environment with cloud computing environment. In this work, different variations of image datasets are taken to find the multi-class disease prediction process. Also, in this work a novel segmentation-based classification model is proposed in order to remove the over-segmentation process on different feature space. In our work, Amazon AWS cloud server with multiple GPU instances are used to simulate the results on the image dataset. The performance of proposed system is measured using specificity (Spec), sensitivity (Sen), accuracy (Acc), and true positive value (PPV).

$$
\begin{gathered}
\text { Sen }=\frac{T P}{(T P+F N)} \\
\text { Spec }=\frac{T N}{(T N+F P)} \\
\mathrm{PPV}=\frac{T P}{(T P+F P)} \\
\mathrm{Acc}=\frac{(T P+T N)}{(T P+T N+F P+F N)}
\end{gathered}
$$

where,

TP (True Positive): Predicting disease patterns in MA region.

FP (False Positive): Wrongly predicting patterns in non MA region.

TN (True Negative): Correctly predicting disease patterns in Non-MA regions

FN (False Negative): Wrongly predicting patterns in nonMA regions.

In this work, different statistical performance metrics are used to find the best solutions for the disease prediction. Along with these four metrics, the runtime of the model is computed to each phase to provide the efficiency of the proposed model than the conventional models.

Table 1 describes the performance of the proposed normalized filtering model to the conventional models on large test dataset. From the figure, it is noted that the present filtered approach has better image denoising than the conventional approaches. In this table, as the number of training images are increasing in size, traditional models require static initialization parameters in order to filter the number of essential key features during the disease prediction process. However, in this model as the number of training images are increasing, proposed model efficiently finds the key features by eliminating the noise in each image for classification process.

Figure 4 illustrates the different types of disease class variation in the input image database. As shown in the figure, each image has different variation in the disease class prediction.

Figure 5 illustrates the normalization process on the input image database for disease prediction process. Figure 5 describes the performance of the proposed normalized filtering model runtime (ms) to the conventional models on large test dataset. From the figure, it is noted that the present filtered approach has better runtime (ms) than the conventional approaches.

Figure 6 illustrates the segmented image features -on the filtered data for disease prediction process.

Figure 7 describes the performance of the proposed normalized filtering based segmentation runtime (ms) to the conventional models on large test dataset. From the figure, it is noted that the present filtered approach has better runtime (ms) than the conventional approaches.
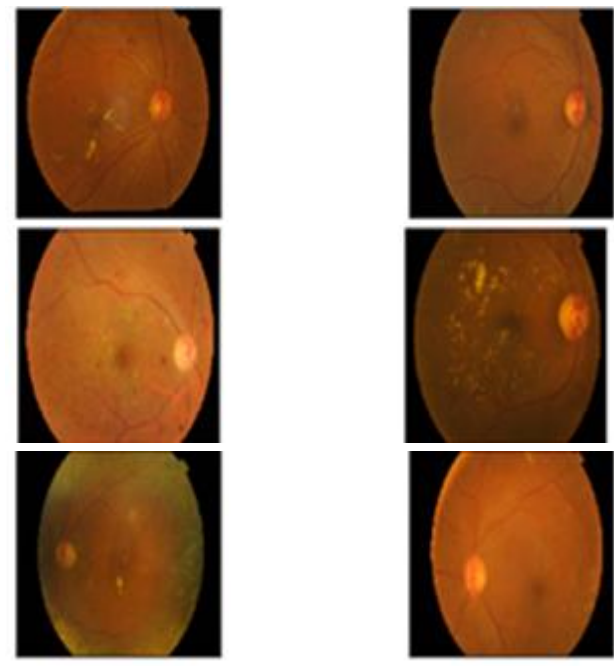

\begin{tabular}{|c|c|c|c|c|}
\hline TestImage & Guasssian & NaiveThreshold & MedianFilter & ProposedFilter \\
\hline \#5 & 42 & 55 & 75 & 83 \\
\hline$\# 10$ & 62 & 60 & 53 & 79 \\
\hline$\# 15$ & 49 & 55 & 66 & 79 \\
\hline$\# 20$ & 44 & 61 & 73 & 79 \\
\hline$\# 25$ & 41 & 53 & 53 & 82 \\
\hline$\# 30$ & 60 & 58 & 68 & 81 \\
\hline$\# 35$ & 47 & 55 & 69 & 78 \\
\hline$\# 40$ & 36 & 53 & 74 & 82 \\
\hline$\# 45$ & 58 & 63 & 71 & 79 \\
\hline \#50 & 54 & 59 & 51 & 82 \\
\hline$\# 55$ & 49 & 53 & 64 & 80 \\
\hline$\# 60$ & 44 & 58 & 55 & 81 \\
\hline$\# 65$ & 55 & 53 & 70 & 81 \\
\hline \#70 & 57 & 60 & 70 & 78 \\
\hline \#75 & 32 & 58 & 62 & 80 \\
\hline$\# 80$ & 43 & 54 & 52 & 78 \\
\hline$\# 85$ & 30 & 58 & 55 & 82 \\
\hline$\# 90$ & 60 & 52 & 58 & 78 \\
\hline$\# 95$ & 33 & 50 & 61 & 83 \\
\hline
\end{tabular}

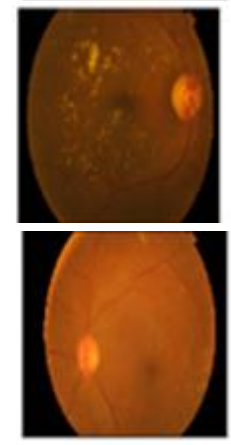

Figure 4. Input image dataset with different class variations

Table 1. Comparative analysis of proposed filtered model than the conventional approaches 

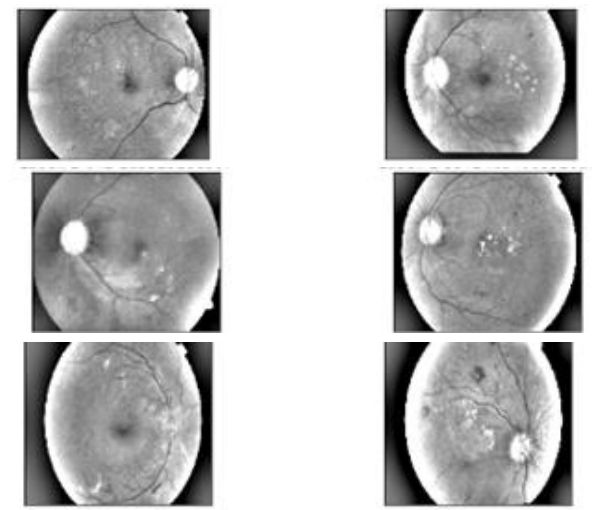

Figure 5. Image thresholding based normalization for segmentation process
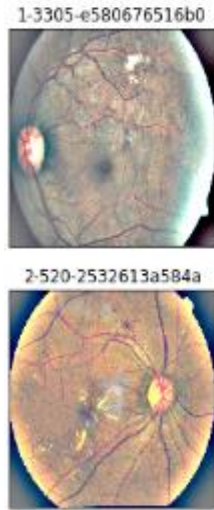

3-2200-9a94e0316ee
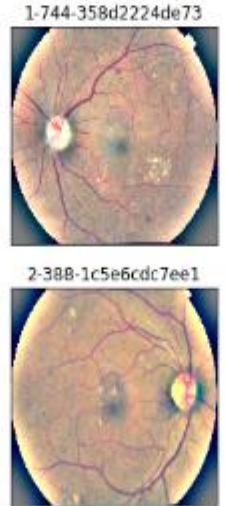

3-1971-Bagbef2fbd4e
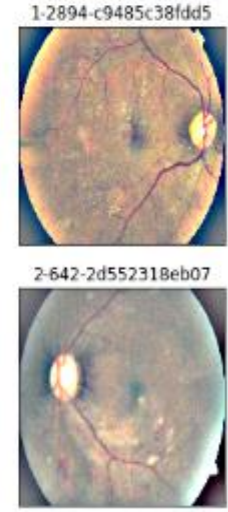

3-1774-7d3835e4e63a
Figure 6. Image segmentation process on the filtered image data

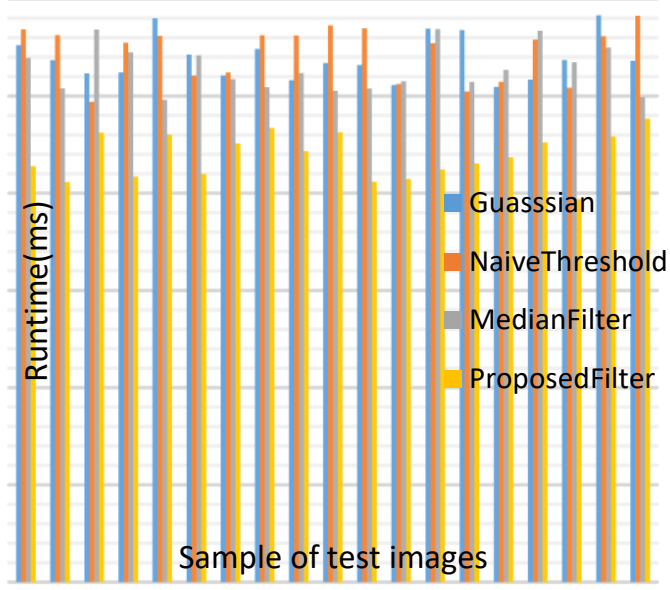

Figure 7. Comparative analysis of proposed filtered model than the conventional approaches

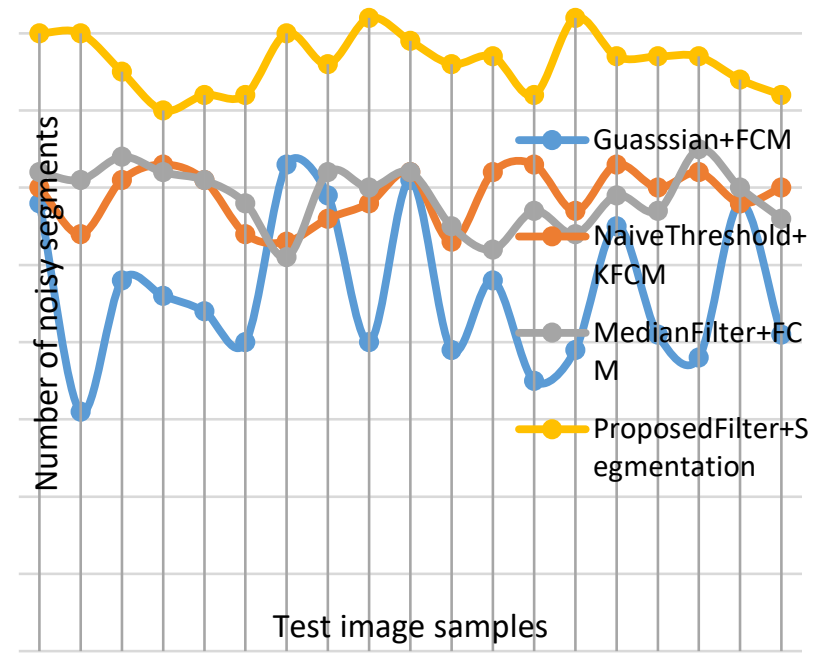

Figure 8. Comparison of proposed filter based segmentation model to traditional model for over segmented regions detection

Figure 8 describes the comparative analysis of proposed approach to the conventional approaches for disease prediction accuracy on input disease database.

Table 2 describes the performance of the proposed normalized filtering based segmentation runtime (ms) to the conventional models on large test dataset. From the figure, it is noted that the proposed filtered approach has better runtime (ms) than the conventional models. In this table, the runtime of each phase of the feature pre-processing and segmentation process are computed in order to provide the fastness of the model as compared to the conventional models.

Table 3 illustrates the comparative analysis of proposed Bayesian deep neural network model than the conventional approaches in terms of true positive rate. From the proposed model, it is noted that the present model has better true positive rate for class prediction than the conventional approaches.

Table 4 describes the performance of the proposed normalized filtering based segmentation and $\mathrm{CNN}$ framework runtime (ms) to the conventional models on training data. From the figure, it is noted that the present model has better runtime (ms) than the conventional approaches. In this table, the runtime of each phase of the feature pre-processing, segmentation and CNN framework are computed in order to provide the fastness of the model as compared to the conventional models. Here, the proposed model is compared with the traditional three efficient models for overall runtime (ms) computation.

Table 2. Comparative analysis of proposed filtered based segmentation model runtime (ms) than the conventional approaches

\begin{tabular}{|c|c|c|c|c|}
\hline TestImage & Guasssian+FCM & NaiveThreshold+KFCM & MedianFilter+FCM & ProposedFilter+Segmentation \\
\hline \#5 & 5961 & 6675 & 6564 & 5058 \\
\hline$\# 10$ & 7207 & 7355 & 7386 & 5302 \\
\hline$\# 15$ & 6145 & 6361 & 6097 & 5240 \\
\hline$\# 20$ & 7070 & 6399 & 6539 & 4924 \\
\hline$\# \mathbf{\# 5}$ & 6050 & 7124 & 7214 & 5367 \\
\hline$\# 30$ & 7006 & 6934 & 7487 & 5064 \\
\hline$\# 35$ & 7469 & 7025 & 6300 & 5033 \\
\hline
\end{tabular}




\begin{tabular}{|c|c|c|c|c|}
\hline TestImage & Guasssian+FCM & NaiveThreshold+KFCM & MedianFilter+FCM & ProposedFilter+Segmentation \\
\hline $\mathbf{\# 4 0}$ & 6758 & 7023 & 6508 & 5051 \\
\hline $\mathbf{4 5}$ & 7164 & 6279 & 7211 & 5276 \\
\hline $\mathbf{\# 5 0}$ & 7333 & 6828 & 7336 & 4919 \\
\hline $\mathbf{\# 5}$ & 6655 & 6595 & 6464 & 5319 \\
\hline $\mathbf{\# 0}$ & 6428 & 6680 & 6159 & 5219 \\
\hline $\mathbf{\# 6 5}$ & 6668 & 7422 & 7353 & 5281 \\
\hline $\mathbf{\# 0}$ & 7238 & 7165 & 6281 & 5275 \\
\hline $\mathbf{\# 5}$ & 7685 & 6578 & 7605 & 5439 \\
\hline $\mathbf{\# 0}$ & 7200 & 7551 & 7312 & 5261 \\
\hline $\mathbf{\# 8 5}$ & 6212 & 7545 & 6351 & 5146 \\
\hline $\mathbf{\# 9}$ & 5954 & 6947 & 7295 & 5262 \\
\hline $\mathbf{\# 5}$ & 7253 & 7648 & 6383 & 5213 \\
\hline
\end{tabular}

Table 3. Comparative analysis of proposed Bayesian deep neural network model than the conventional approaches

\begin{tabular}{|c|c|c|c|c|}
\hline TestImage & G+FCM+SVM & NT+KFCM+SVM & MF+FCM+SVM & ProposedBNN \\
\hline $\mathbf{\# 5}$ & 0.92 & 0.86 & 0.87 & 0.96 \\
\hline $\mathbf{\# 1 0}$ & 0.92 & 0.88 & 0.87 & 0.96 \\
\hline $\mathbf{\# 1 5}$ & 0.92 & 0.86 & 0.86 & 0.95 \\
\hline $\mathbf{\# 0}$ & 0.86 & 0.9 & 0.88 & 0.98 \\
\hline $\mathbf{\# 2 5}$ & 0.88 & 0.91 & 0.87 & 0.97 \\
\hline $\mathbf{\# 3}$ & 0.93 & 0.85 & 0.9 & 0.95 \\
\hline $\mathbf{\# 3}$ & 0.86 & 0.86 & 0.93 & 0.97 \\
\hline $\mathbf{\# 4 5}$ & 0.85 & 0.9 & 0.92 & 0.96 \\
\hline $\mathbf{\# 5}$ & 0.87 & 0.86 & 0.91 & 0.97 \\
\hline $\mathbf{\# 5}$ & 0.88 & 0.92 & 0.92 & 0.96 \\
\hline $\mathbf{\# 6 5}$ & 0.94 & 0.89 & 0.93 & 0.96 \\
\hline $\mathbf{\# 0}$ & 0.89 & 0.92 & 0.94 & 0.97 \\
\hline $\mathbf{\# 5}$ & 0.86 & 0.88 & 0.94 & 0.97 \\
\hline $\mathbf{\# 0}$ & 0.9 & 0.9 & 0.89 & 0.96 \\
\hline $\mathbf{\# 5}$ & 0.87 & 0.89 & 0.86 & 0.96 \\
\hline $\mathbf{\# 0}$ & 0.86 & 0.93 & 0.87 & 0.97 \\
\hline
\end{tabular}

Table 4. Comparative analysis of proposed Bayesian deep neural network model than the conventional approaches for runtime (ms) computation

\begin{tabular}{|c|c|c|c|c|}
\hline TestImage & G+FCM+SVM & NT+KFCM+SVM & $\mathrm{MF}+\mathrm{FCM}+\mathrm{SVM}$ & ProposedBNN \\
\hline \#5 & 9292.72 & 8302.77 & 8941.64 & 7194.96 \\
\hline$\# 10$ & 9489.26 & 8094.93 & 9254.64 & 7532.4 \\
\hline$\# 15$ & 9047.76 & 7994.98 & 9192.58 & 7353.04 \\
\hline$\# 20$ & 8983.53 & 8530.68 & 8334.79 & 6637.25 \\
\hline$\# 25$ & 9663.23 & 8914.66 & 9129.48 & 7260.05 \\
\hline \#30 & 8437.84 & 8009.39 & 8807.82 & 7359.07 \\
\hline \#35 & 9552.05 & 8140.2 & 9605.81 & 7369.75 \\
\hline \#40 & 8110.05 & 8512.82 & 8265.67 & 7641.25 \\
\hline \#45 & 9094.75 & 8377.68 & 8410.4 & 6980.46 \\
\hline \#50 & 9439.55 & 8927.82 & 9642.13 & 6839.56 \\
\hline \#55 & 9437.99 & 9821.56 & 9005.41 & 6816.75 \\
\hline \#60 & 8747.03 & 8884.61 & 9281.93 & 7260.46 \\
\hline \#65 & 9395.03 & 9169.29 & 8843.24 & 6545.16 \\
\hline \#70 & 8544.49 & 8530.8 & 8381.39 & 7312.29 \\
\hline \#75 & 9183.64 & 9122.24 & 9366.49 & 7415.86 \\
\hline$\# 80$ & 9828.8 & 9711.06 & 8442.55 & 7009.56 \\
\hline \#85 & 9312.21 & 7980.27 & 8570.35 & 7390.96 \\
\hline \#90 & 8106.63 & 9297.25 & 8857.46 & 7555.64 \\
\hline \#95 & 8599.36 & 9712.53 & 9681.72 & 7621.14 \\
\hline
\end{tabular}

\section{CONCLUSIONS}

In this paper, a novel Bayesian deep learning framework is designed and implemented on the large Diabetic retinopathy databases. Since, most of the traditional Diabetic retinopathy detection models are depend on single variation in the feature space, it is difficult to process different types of DR class variation due to over-segmented features and dimensions. In this work, a novel image filtering approach, optimal image segmentation approach and hybrid Bayesian deep learning 
framework are developed on the large DR image databases. In this paper, a hybrid thresholding based segmentation algorithm and classification model are developed in order to improve the efficiency of the model for disease prediction. Experimental results proved that the proposed filtered segmentation-based Bayesian deep neural network has better accuracy $(5 \%)$ and runtime $(\mathrm{ms})(3 \%)$ than the conventional models on different DR variation databases. In future work, this model is extended to non-linear classification model in order to improve the error rate and true positive rate of the noisy test samples.

\section{REFERENCES}

[1] Akram, M.U., Akbar, S., Hassan, T., Khawaja, S.G., Yasin, U., Basit, I. (2020). Data on fundus images for vessels segmentation, detection of hypertensive retinopathy, diabetic retinopathy and papilledema. Data in $\quad$ Brief, 105282. http://dx.doi.org/10.1016/j.dib.2020.105282

[2] Asiri, N., Hussain, M., Al Adel, F., Alzaidi, N. (2019). Deep learning based computer-aided diagnosis systems for diabetic retinopathy: A survey. Artificial Intelligence in Medicine, 99: 101701. http://dx.doi.org/10.1016/j.artmed.2019.07.009

[3] Devaraj, D., Suma, R., Prasanna Kumar, S.C. (2018). A survey on segmentation of exudates and microaneurysms for early detection of diabetic retinopathy. Materials Today: Proceedings, 5(4): 10845-10850. http://dx.doi.org/10.1016/j.matpr.2017.12.372

[4] Franklin, S.W., Rajan, S.E. (2014). Computerized screening of diabetic retinopathy employing blood vessel segmentation in retinal images. Biocybernetics and Biomedical Engineering, 34(2): 117-124. http://dx.doi.org/10.1016/j.bbe.2014.01.004

[5] Jebaseeli, T.J., Deva Durai, C.A., Peter, J.D. (2019). Retinal blood vessel segmentation from diabetic retinopathy images using tandem PCNN model and deep learning based SVM. Optik, 199: 163328. http://dx.doi.org/10.1016/j.ijleo.2019.163328

[6] Jebaseeli, T.J., Durai, C.A.D., Peter, J.D. (2019). Segmentation of retinal blood vessels from ophthalmologic Diabetic Retinopathy images. Computers \& Electrical Engineering, 73: 245-258. http://dx.doi.org/10.1016/j.compeleceng.2018.11.024

[7] Köse, C., Şevik, U., İkibaş, C., Erdöl, H. (2012). Simple methods for segmentation and measurement of diabetic retinopathy lesions in retinal fundus images. Computer Methods and Programs in Biomedicine, 107(2): 274-293. http://dx.doi.org/10.1016/j.cmpb.2011.06.007

[8] Li, T., Gao, Y., Wang, K., Guo, S., Liu, H., Kang, H. (2019). Diagnostic assessment of deep learning algorithms for diabetic retinopathy screening. Information Sciences, 501: 511-522. http://dx.doi.org/10.1016/j.ins.2019.06.011

[9] Mirshahi, A., Ghassemi, F., Fadakar, K., Mirshahi, R., Bazvand, F., Riazi-Esfahani, H. (2019). Effects of panretinal photocoagulation on retinal vasculature and foveal avascular zone in diabetic retinopathy using optical coherence tomography angiography: A pilot study. Journal of Current Ophthalmology, 31(3): 287291. http://dx.doi.org/10.1016/j.joco.2019.06.001

[10] Porwal, P., Pachade, S., Kokare, M., et al. (2020). IDRiD:
Diabetic Retinopathy - Segmentation and Grading Challenge. Medical Image Analysis, 59: 101561. http://dx.doi.org/10.1016/j.media.2019.101561

[11] Sadda, S.R., Nittala, M.G., Taweebanjongsin, W., Verma, A., Velaga, S.B., Alagorie, A.R., Sears, C.M., Silva, P.S., Aiello, L.P. (2020). Quantitative assessment of the severity of diabetic retinopathy. American Journal of Ophthalmology, 218: 342-352. http://dx.doi.org/10.1016/j.ajo.2020.05.021

[12] Salamat, N., Missen, M.M.S., Rashid, A. (2019). Diabetic retinopathy techniques in retinal images: A review. Artificial Intelligence in Medicine, 97: 168-188. http://dx.doi.org/10.1016/j.artmed.2018.10.009

[13] Samanta, A., Saha, A., Satapathy, S.C., Fernandes, S.L., Zhang, Y.D. (2020). Automated detection of diabetic retinopathy using convolutional neural networks on a small dataset. Pattern Recognition Letters, 135: 293-298. http://dx.doi.org/10.1016/j.patrec.2020.04.026

[14] Sambyal, N., Saini, P., Syal, R., Gupta, V. (2020). Modified U-Net architecture for semantic segmentation of diabetic retinopathy images. Biocybernetics and Biomedical Engineering, 40(3): 1094-1109. http://dx.doi.org/10.1016/j.bbe.2020.05.006

[15] Selçuk, T., Alkan, A. (2019). Detection of microaneurysms using ant colony algorithm in the early diagnosis of diabetic retinopathy. Medical Hypotheses, 129: 109242. http://dx.doi.org/10.1016/j.mehy.2019.109242

[16] Shankar, K., Sait, A.R.W., Gupta, D., Lakshmanaprabu, S.K., Khanna, A., Pandey, H.M. (2020). Automated detection and classification of fundus diabetic retinopathy images using synergic deep learning model. Pattern Recognition Letters, 133: 210-216. http://dx.doi.org/10.1016/j.patrec.2020.02.026

[17] Stolte, S., Fang, R. (2020). A survey on medical image analysis in diabetic retinopathy. Medical Image Analysis, 64: http://dx.doi.org/10.1016/j.media.2020.101742 101742.

[18] Waqar, S., Bullen, G., Chant, S., Salman, R., Vaidya, B., Ling, R. (2012). Cost implications, deprivation and geodemographic segmentation analysis of non-attenders (DNA) in an established diabetic retinopathy screening programme. Diabetes \& Metabolic Syndrome: Clinical Research \& Reviews, 6(4): 199-202. http://dx.doi.org/10.1016/j.dsx.2012.08.009

[19] Washburn, P.S., Mahendran, Dhanasekharan, Periyasamy, Murugeswari. (2020). Investigation of severity level of diabetic retinopathy using adaboost classifier algorithm. Materials Today: Proceedings, 33: 3037-3042. http://dx.doi.org/10.1016/j.matpr.2020.03.199

[20] Xie, Q., Liu, Y., Huang, H., Hong, B., Wang, J., Han, H., Liu, Y. (2020). An innovative method for screening and evaluating the degree of diabetic retinopathy and drug treatment based on artificial intelligence algorithms. Pharmacological Research, 159: 104986. http://dx.doi.org/10.1016/j.phrs.2020.104986

[21] Xue, J., Yan, S., Qu, J., Qi, F., Qiu, C., Zhang, H., Chen, M., Liu, T., Li, D., Liu, X. (2019). Deep membrane systems for multitask segmentation in diabetic retinopathy. Knowledge-Based Systems, 183: 104887. http://dx.doi.org/10.1016/j.knosys.2019.104887

[22] You, Q.S., Wang, J., Guo, Y., Pi, S., Flaxel, C.J., Bailey, S.T., Huang, D., Jia, Y., Hwang, T.S. (2020). Optical 
coherence tomography angiography avascular area association with one-year treatment requirement and disease progression in diabetic retinopathy. American Journal of Ophthalmology, 2017: 268-277. http://dx.doi.org/10.1016/j.ajo.2020.04.024

[23] Amin, J., Sharif, M., Yasmin, M., Ali, H., Fernandes, S.L. (2017). A method for the detection and classification of diabetic retinopathy using structural predictors of bright lesions. Journal of Computational Science, 19: 153-164. http://dx.doi.org/10.1016/j.jocs.2017.01.002

[24] de la Torre, J., Valls, A., Puig, D. (2020). A deep learning interpretable classifier for diabetic retinopathy disease grading. Neurocomputing, 396: 465-476. http://dx.doi.org/10.1016/j.neucom.2018.07.102

[25] Kamble, V.V., Kokate, R.D. (2020). Automated diabetic retinopathy detection using radial basis function. Procedia Computer Science, 167: 799-808. http://dx.doi.org/ 10.1016/j.procs.2020.03.429

[26] Kumar, S., Adarsh, A., Kumar, B., Singh, A.K. (2020).
An automated early diabetic retinopathy detection through improved blood vessel and optic disc segmentation. Optics \& Laser Technology, 121: 105815. http://dx.doi.org/10.1016/j.optlastec.2019.105815

[27] Liu, Y.P., Li, Z., Xu, C., Li, J., Liang, R. (2019). Referable diabetic retinopathy identification from eye fundus images with weighted path for convolutional neural network. Artificial Intelligence in Medicine, 99: 101694. http://dx.doi.org/10.1016/j.artmed.2019.07.002

[28] Shanthi, T., Sabeenian, R.S. (2019). Modified Alexnet architecture for classification of diabetic retinopathy images. Computers \& Electrical Engineering, 76: 56-64. http://dx.doi.org/10.1016/j.compeleceng.2019.03.004

[29] Wang, F., Saraf, S.S., Zhang, Q., Wang, R.K., Rezaei, K.A. (2020). Ultra-widefield protocol enhances automated classification of diabetic retinopathy severity with OCT angiography. Ophthalmology Retina, 4(4): 415-424. http://dx.doi.org/10.1016/j.oret.2019.10.018 